\title{
DIVISION I / WORKING GROUP NOMENCLATURE FOR FUNDAMENTAL ASTRONOMY
}

\author{
PRESIDENT \\ MEMBERS
}

\author{
Nicole Capitaine \\ Alexandre H. Andrei, Mark R. Calabretta, \\ Véronique Dehant, Toshio Fukushima, \\ Bernard R. Guinot, Catherine Y. Hohenkerk, \\ George H. Kaplan, Sergei A. Klioner, \\ Jean Kovalevsky, Irina I. Kumkova, \\ Chopo Ma, Dennis D. McCarthy, \\ P. Kenneth Seidelmann, Patrick T. Wallace
}

\section{PROCEEDINGS BUSINESS MEETING on 16 August 2006}

\section{Introduction}

The IAU Division I Working Group on Nomenclature for Fundamental Astronomy (NFA) was established by the IAU XXV General Assembly with the task of providing proposals for new nomenclature associated with the implementation of the IAU XXIV GA resolutions (2000) and to make related educational efforts for bringing the issue to the notice of scientists in the community.

A meeting of this working group was organized during the IAU XXVI GA in order to adopt the final conclusions of the WG and to discuss the status of the WG resolutions proposals to the IAU before they were discussed at the Division I meetings and then submitted to the IAU GA at its second meeting (24 August). This WG meeting was also an opportunity to discuss the final scheme and content of the NFA explanatory document, as well as the plan for publication of the NFA recommendations and of the NFA explanatory document. The future diffusion of the NFA educational documents was also discussed. Finally, a list of scientific issues were identified by the NFA WG to be proposed to Division I as topics for its proposed new WGs. Ten out of fifteen WG members participated in this NFA WG meeting. A few other participants, not belonging to the WG, were invited to attend the meeting, including the upcoming Division I president, Jan Vondrák.

\section{The NFA Working Group recommendations on terminology}

At the time the WG was established, the series of resolutions on reference systems adopted by the IAU XXIV GA in 2000 (i.e. B1.1 to B1.9) had just been implemented in the IERS Conventions (2003) and SOFA 2003 release (Wallace 2004). Several terminology issues were identified during the Division I meetings at the IAU XXV GA (see IAU Transactions XXVB, 2008), which required further studies and discussions among experts.

During the period 2003-2006, the NFA Working Group has worked on selecting a consistent and well defined terminology for all the quantities based on the IAU XXIV GA (2000) resolutions in order that it will be understood, recognized and adopted by the astronomical community. The terminology and guidelines recommended by the WG have been prepared through WG e-mail discussion and have been described in a number of Newsletters and documents. All the WG documents have been regularly posted on the NFA web site (at: <http://syrte.obspm.fr/iauWGnfa/>), which includes links to other sites relevant to the WG activities. A special page of the web site provides links to educational documents that explain the NFA issues. Two open WG discussions of about 100 participants were organized during the Journées 2004 and 2005 in Paris and Warsaw, respectively, where successive drafts of NFA recommendations were submitted for comment. 
There has been detailed WG discussion on the terminology choices related to IAU XXIV GA (2000) resolutions B1.3, B1.5, B1.6, B1.7 and B1.8 with coordination with the International Association of Geodesy for the terrestrial reference system definitions.

The final WG recommendations on terminology that have been adopted are the following:

1. Using existing terms (e.g., right ascension) in extended ways for the terminology associated with the new paradigm with a clear specification, rather than introducing new names.

2. Using "equinox based" and "CIO based" for referring to the classical and new paradigms, respectively.

Comment: the "Celestial/Terrestrial Intermediate Origin" with the acronym CIO/TIO is proposed here as the updated terminology to replace the IAU XXIV GA (2000) "Celestial/Terrestrial Ephemeris Origin" with the acronym CEO/TEO (see below items 3 and 4 and the proposed resolution).

3. Using "intermediate" to describe (i) the moving geocentric celestial reference system defined in the IAU 2000 resolutions (i.e. containing the CIP and the CIO), and (ii) the moving terrestrial system containing the CIP and the TIO.

Comment: the term "intermediate" has been chosen to specify that these systems are intermediary systems between the geocentric celestial system and the terrestrial system, which are realized by using the models, constants and procedures that are conventionally accepted; it conventionally separates the instantaneous celestial orientation of the Earth into components we label polar motion (in the terrestrial system) and precession-nutation (in the celestial system).

4. Harmonizing the name of the pole and the origin to "intermediate" and therefore changing CEO/TEO to CIO/TIO.

5. Using "system" in a broad sense rather than "frame" in this context of the intermediary system/frame.

6. Using special designations for particular realizations of the intermediate celestial system.

Comment: this applies for example to "the IAU 2000A system" to designate the system which is realized by transforming the geocentric celestial system GCRS to the intermediate system using the IAU 2000A precession-nutation and associated frame biases at J2000 (the GCRS being transformed from the BCRS by using the coordinate transformation specified in the IAU 2000 Resolution B1.3).

7. Keeping the classical terminology for "true equator and equinox" (or "true equinox based") for the classical equatorial system.

8. Choosing "equinox right ascension" (or "RA with respect to the equinox") and "intermediate right ascension" (or "CIO right ascension", or "RA with respect to the CIO"), for the azimuthal coordinate along the equator in the classical and new paradigms, respectively. (Note that right ascensions and declinations with respect to the ICRS are usually designated by $\left.\alpha_{I C R S}, \delta_{I C R S}\right)$. Comment: this is to be specified only once in the presentation of a paper if there is some risk of misunderstanding. Afterward, "right ascension" alone is sufficient.

9. Giving the name "equation of the origins" to the distance between the CIO and the equinox along the intermediate equator, the sign of this quantity being such that it represents the CIO right ascension of the equinox, or equivalently, the difference between the Earth Rotation Angle and Greenwich apparent sidereal time.

10. Retaining "apparent places" and "mean places" in the equinox based system.

11. Not introducing "apparent intermediate places" in the CIO based system, but introducing instead "intermediate places".

12. Using "ITRF zero-meridian" to designate the plane passing through the geocenter, ITRF pole and ITRF $x$-origin and using, if necessary, "TIO meridian" to designate the moving plane passing through the geocenter, the CIP and the TIO. 
13. Fixing the default orientation of the BCRS so that for all practical applications, unless otherwise stated, the BCRS is assumed to be oriented according to the ICRS axes.

Comment: Once the BCRS is spatially oriented according to the ICRS, the spatial GCRS coordinates get an "ICRS-induced" orientation.

14. Re-defining Barycentric Dynamical Time (TDB) as a fixed linear function of TCB:

$\mathrm{TDB}=\mathrm{TCB}-\mathrm{L}_{\mathrm{B}} \times\left(\mathrm{JD}_{\mathrm{TCB}}-\mathrm{T}_{0}\right) \times 86400+\mathrm{TDB}_{0}$,

where $\mathrm{T}_{0}=2443144.5003725$,

and $\mathrm{L}_{\mathrm{B}}=1.550519768 \times 10^{-8}$ and $\mathrm{TDB}_{0}=-6.55 \times 10^{-5} \mathrm{~s}$ are defining constants.

Additional points

- Considering a terminology associated with other types of apparent places, although it may be required for specific use, has not been considered as being essential for common astronomical use and is therefore not part of the NFA WG terminology recommendations.

- No WG consensus having been reached for having strict rules for using or not using capitals for names for origins, poles and systems, no recommendation on this issue is proposed by the $W G$. The policy adopted throughout the NFA document is to capitalize those terms that are defined in IAU or IUGG resolutions.

\section{The NFA resolution proposals to the IAU}

The major NFA Recommendations have resulted in two IAU resolution proposals. The first one recommends 1) using the designation "intermediate" to describe the moving celestial and terrestrial reference systems defined in the IAU 2000 resolutions and the various related entities, and 2) fixing the default orientation of the Barycentric and Geocentric celestial reference systems GCRS to that of the ICRS axes. The second one recommends a re-definition of Barycentric Dynamical time (TDB).

Successive versions of the resolution proposals were discussed by the WG during the period 2004-2006 and posted on the NFA WG web page. In March 2006, the final wording of the NFA resolution proposals was adopted by the NFA WG, in coordination with the IAU Working Group on "Relativity in Celestial Mechanics, Astrometry and Metrology", chaired by M. Soffel, and also with G. Petit and M. Standish. The proposals were submitted to Commissions 4, 8, 19, 31 for approval, and then supported by Division 1. In April 2006, the NFA resolutions proposals were officially submitted to the IAU. In July 2006, the resolutions to be presented for voting at the IAU General Assembly, including those proposed by the NFA WG, were posted on the IAU web site and provided in IAU Newsletter 5 . They were published in the official GA newspaper of 16 August 2006. The IAU resolutions were submitted to be voted on during the second session of the 26th IAU GA.

The status of the resolution proposals was discussed during the WG meeting in order to decide on the process for further discussion before it was voted by the IAU GA. The comments on the NFA proposals that had been received were discussed and a few changes were proposed to the wording in order to address the concerns that had been expressed. It was agreed that the revised versions should be presented at Division 1 meetings during the GA.

[The two resolutions submitted to the Executive Committee by the Working Group were voted on at the final General Assembly meeting on 2006 August 24. Both resolutions were adopted. They are resolutions 2 and 3 from the XXVIth General Assembly.]

\section{The NFA explanatory document}

The content of the NFA Explanatory document was presented and discussed. The introduction should present the background on the IAU 2000 resolutions on reference systems and a summary of the terminology work. Part A should review the basis of the IAU resolutions and their implementation. Part B should contain the "NFA IAU 2000 Glossary", which provides a set of detailed definitions that best explain all the terms required for implementing the IAU 2000 resolutions, including a few newly proposed terms. It is completed by additional documents including a chart, the purpose of which is to illustrate the various stages showing the BCRSto-GCRS-to-ITRS transformation in General relativity (Resolution B1.3) and the parallel CIO and equinox based processes (Resolution B1.8). 
The following scheme has been adopted:

- Introduction to the document

- Part A: Basis of the IAU resolutions and their implementation

Historical review: reasons for the changes

Detailed explanations on Resolutions B1.3, B1.5, B1.6, B1.7, B1.8, B1.9

Description of the new paradigm and comparison with the classical one

Detailed discussion on a few issues especially discussed by the WG

- Part B: Explanation of the proposed terminology

NFA IAU 2000 Glossary

Table containing the categorized list of terms

Chart of the transformation process from ICRS to observed places of stars

List of abbreviations, acronyms and symbols

- References

Part B of the NFA Explanatory document is already realized, but the other parts of the document have still to be done.

Plans for the future work were discussed. Due to the current rules of the scientific organization of the IAU, this working group, which has met its objectives within the three-year period, should be disbanded at the end of this GA. Hence the WG document will need to be finalized outside the official IAU WG framework.

\section{Plan for publication of the NFA working group documents}

Preliminary reports of the NFA WG has been published (IAU Transactions A 2006, Capitaine et al. 2005, 2006), and the report of this WG meeting will be part of the Proceedings of the XXVIth IAU GA.

The publication of the final NFA WG document, consisting of the recommendations and the explanatory material, was identified as an important issue and several options were considered during the meeting. The meeting concluded that the final document should be published, both electronically and in a referred journal. However, due to the document size and the fact that it deals with terminology issues, it was recognized that it may not be accepted in any of the journals in which scientific papers in fundamental astronomy are published (e.g. $A J, A \& A$, or Celest. Mech. Dyn. Astr.). If this occurs, then other options for its publication needs to be considered. One possibility, offered by B. Luzum, was to include the NFA Glossary (part of the final NFA document) in the next release of the IERS Conventions.

\section{Plan for diffusion of the NFA educational documents}

For educational purposes, a number of presentations made by members of the NFA WG have been realized as PDF files, and are currently on line on the NFA WG website. They include the following:

- The IAU Recommendations on Reference Systems and their applications

- Recent International Recommendations on Reference Systems

- SOFA software support for IAU 2000

- The ICRS, BCRS and GCRS and the ITRS

- Recent progress in astronomical nomenclature in the relativistic framework

- Progress on the implementation of the new nomenclature in "The Astronomical Almanac"

- Latest proposals of the IAU Working Group on Nomenclature for fundamental astronomy

- Développements récents des concepts et des modèles en Astronomie fondamentale

- 3D representation of the Non-Rotating Origin (with movies)

Additionally, an example transformation (by P. Wallace) is currently on line on the NFA WG web site providing an application of the IAU 2000 resolutions concerning Earth orientation and rotation, with the objective of predicting the topocentric apparent direction of a star.

The plan for diffusion of those educational documents has been discussed. Mirror sites may be developed which will include all the files and thus increase accessibility. Other options considered were depositing the files with the CDS or making them available on the IAU website. 


\section{Concluding remarks}

The NFA WG, created at the 2003 IAU General Assembly, has reached its aim with selecting a consistent and well defined terminology for all the quantities based on the IAU 2000 resolutions on reference systems. The conclusion of this work have been reflected in 14 recommendations, which are supported by two explanatory documents and educational documents.

It should be noted that the NFA recommendations on terminology have already been taken into account in the 2006 editions of the Almanac offices that have implemented the IAU 2000 resolutions (Hohenkerk 2005), in the updated IERS Conventions (ongoing), and USNO Circular 179 (Kaplan 2005).

The NFA Recommendations have resulted into two IAU resolution proposals that have been labeled as IAU 2006 Resolutions 2 and 3. [Both resolutions were adopted by the GA.]

The publication of the final NFA WG recommendations and of the NFA explanatory document has to be realized in the most appropriate form. It will be finalized outside the official IAU WG framework.

The scientific issues identified by the NFA WG to be proposed to Division 1 as topics for a new WG or Commission:

- TDB units, astronomical units,

- appropriate nomenclature and description for topocentric reference systems,

- definition of the ecliptic within the GCRS,

- various effects in the dynamics of the rotating Earth and new theories,

- astronomical constants,

- coordination between IERS Conventions and SOFA.

The work accomplished by the NFA WG work has been very fruitful with recommendations that are already followed in recent publications and two resolutions proposed to the IAU. This has been the work of the whole working group and it is my pleasure to thank all the WG members for their efforts and efficient cooperation during this 3-year undertaking.

$$
\begin{array}{r}
\text { Nicole Capitaine } \\
\text { chair of the Working Group }
\end{array}
$$

\section{References}

Capitaine, N., Hohenkerk, C., Andrei, A. H., Calabretta, M., Dehant, V., Fukushima, T., Guinot, B., Kaplan, G., Klioner, S., Kovalevsky, J., Kumkova, I., Ma, C., Mccarthy, D. D., Seidelmann, K., \& Wallace, P. 2005, in Proceedings of the "Journées 2004 Systèmes de référence spatio-temporels," N. Capitaine (ed), Observatoire de Paris, p. 161

Capitaine, N., Hohenkerk, C., Andrei, A. H., Calabretta, M., Dehant, V., Fukushima, T., Guinot, B., Kaplan, G., Klioner, S., Kovalevsky, J., Kumkova, I., Ma, C., Mccarthy, D. D., Seidelmann, K., \& Wallace, P. 2006, in Proceedings of the "Journées 2005 Systèmes de référence spatio-temporels," A. Breziński et al. (eds), Warsaw, in press

Hohenkerk, C. 2005, in Proceedings of the "Journées 2004 Systèmes de référence spatiotemporels," N. Capitaine (ed), Observatoire de Paris, 168

IAU Transactions (2003) Vol. XXVB, in "Proceedings of the Twenty-Fifth General Assembly", Sydney, ed. O. Engvold, ASP, 2008

IAU Transactions (2003) Vol. XXVIA, "Reports on Astronomy 2003-2005", ed. O. Engvold, CUP, 2007

IERS Conventions (2003), IERS Technical Note 32, D.D. McCarthy \& G. Petit (eds), Frankfurt am Main: Verlag des Bundesamts für Kartographie und Geodäsie, 2004

Kaplan, G. H. 2005, The IAU Resolutions on Astronomical Reference Systems, Time Scales and Earth Rotation Models, USNO Circular No. 179

Wallace P. T. 2004, SOFA software support for IAU 2000, AAS Meeting 204, \#28.02, May 2004 